\title{
les sollicitations à long terme des revêtements des tunnels
}

\section{long terme loading of tunnel lining}

\author{
G. ROUSSET \\ Laboratoire de Mécanique des Solides ${ }^{\circ}$
}

Rev. Franç. Géotech. n 53, pp. 5-20 (octobre 1990)

\section{Résumé}

Dans le cas de certains ouvrages, même profonds, réalisés dans des matériaux tendres (argiles notamment), les effets différés sont très importants et conditionnent le comportement à long terme de l'ouvrage et donc son dimensionnement.

On présente dans ce texte une méthodologie d'étude de ces effets différés allant des problèmes amont (rhéologie des matériaux) jusqu'au choix et au dimensionnement du soutènement.

L'ensemble de la démarche, axée sur la méthode convergence-confinement, est illustrée sur un cas concret.

\footnotetext{
Abstract

In case of underground constructions in soft rocks (particularly clay) the time dependent aspects are very significant and control the long term behaviour of the construction and therefore the choice of its dimensions.

In this paper, a methodology is presented that forms a broad picture of the problem (from basic rheological experiments to the choice and dimensioning of the excavation lining).

The whole procedure incorporating the convergence confinement method is illustrated for a real case.
} 


\section{INTRODUCTION}

La plupart des ouvrages réalisés en souterrain présentent des effets différés qui peuvent durer de quelques semaines à plusieurs années.

Ces effets différés se manifestent par l'augmentation de la convergence de la paroi du tunnel ainsi que par la croissance progressive de la "pression de confinement», c'est-à-dire de la poussée exercée par les terrains sur le soutènement mis en place.

Ils sont dus essentiellement au comportement mécanique de la roche encaissante et sont d'autant plus marqués que les caractéristiques de fluage de la roche sont importantes (argiles ou marnes en particulier).

Il faut noter également que le soutènement lui-même peut engendrer des effets différés (retrait et fluage du béton par exemple).

Si, par un souci d'économie et de sûreté, lobjectif recherché lors de la construction d'un ouvrage est de limiter la pression qu'il subira à long terme de la part du massif, l'étude de ces effets différés doit être réalisée avec soin.

Cette étude passe nécessairement par une connaissance fine du comportement mécanique de la roche.

Cette étape étant franchie, l'étude peut déboucher sur une optimisation des paramètres fondamentaux qui conditionnent la réalisation de l'ouvrage, c'est-à-dire la vitesse de creusement, le délai de pose du soutènement, la nature et le dimensionnement de ce dernier.

Dans ce texte, on se propose de donner une méthodologie générale pour l'étude de ces comportements différés, méthodologie quî débouche sur des prescriptions techniques pour la réalisation des ouvrages.

D'abord, la méthode convergence-confinement, support de la théorie, est commentée et appliquée au cas des roches à effets différés.

Ensuite, on décrit les lois de comportement de ce type de roches, en donnant quelques indications sur les études expérimentales (de laboratoire ou in situ) nécessaires au calage de paramètres.

L'étape suivante concerne l'étude de l'interaction massif-soutènement et débouche sur une estimation de Pinfluence des paramètres fondamentaux adaptés à chaque cas particulier.

Enfin, l'ensemble de la démarche est illustré sur un cas concret qui concerne une galerie cintrée réalisée à $230 \mathrm{~m}$ de profondeur dans une argile plastique.

\section{MÉTHODE \\ CONVERGENCE-CONFINEMENT}

\subsection{Principes généraux de la méthode (AFTES, 1978, [1])}

La méthode convergence-confinement, par comparaison avec les autres méthodes d'étude de la stabilité des tunnels présente deux avantages majeurs:
- elle décrit l'interaction entre le massif et le soutènement, c'est-à-dire qu'elle étudie l'équilibre entre deux structures distinctes, dont les comportements sont très différents: le massif percé par la galerie d'une part et le soutènement d'autre part;

- elle permet, grâce à un artifice que l'on décrira plus loin, de tenir compte de l'aspect tridimensionnel du problème (avancement du front).

A linverse un inconvénient restreint son champ d'application:

La méthode ne s'applique simplement que si la géo. métrie du tunnel est régulière (géométrie quasicylindrique en pratique) et que le massif encaissant est homogène avec un comportement mécanique et un état de contraintes naturelles isotropes. Si l'une de ces deux conditions n'est pas remplie, la modélisation ne donne que des résultats très incertains.

Rappelons rapidement le principe de la méthode. dans le cas le plus simple d'une géométrie cylindrique et d'une roche homogène et isotrope sans effets différés.

L'ensemble des hypothèses prises permet de décrire l'interaction massif-soutènement au moyen de deux paramètres seulement (Fig. 1):

- la convergence $U_{i}$ de la paroi c'est-à-dire la variation relative du diamètre ou du rayon de l'excavation;

- le confinement $\mathrm{P}_{1}$ c'est-à-dire la pression radiale exercée sur la paroi et sur l'extrados du soutènement. De façon à étudier le problème en condition de déformation plane, on rend compte de l'avancement du front par une pression fictive $\mathrm{P}^{f}$ qui passe progressivement de la valeur $P_{\infty}$ (pression lithostatique) avant creusement à 0 lorsque le front est suffisamment loin de la section d'étude. On écrit en général (PANET et GUELLEC, 1974, [10]) :

$$
P_{f}^{f}(X)=(1-\lambda(X)) P_{\infty}
$$

et l'allure de $\mathrm{P}_{1}^{\prime}$ en fonction de la distance $\mathrm{X}$ de la section d'étude au front est indiquée sur la figure $2^{*}$. Le confinement $P_{1}$ est la somme de cette pression fictive et de la pression de soutènement $\mathrm{P}_{\mathrm{f}}^{\mathrm{f}}$ qui, en pratique est la seule qui soit mesurable.

$$
P_{i}(t)=P_{f}^{f}(t)+P_{i}^{s}(t)
$$

(Si ts est l'instant de pose du soutènement, on convient que $\mathrm{P}_{j}^{\mathrm{s}}(\mathrm{t})=0$ pour $\mathrm{t}<\mathrm{ts}$ )

De même la convergence $U_{i}$ est la somme de la convergence $U^{\circ}$ produite avant la pose du soutènement (due au relâchement des terrains) et de la convergence $U_{j}^{s}$ du soutènement:

$$
\begin{aligned}
& U_{i}(t)=U_{i}^{\circ}(t)+U_{1}^{s}(t) \\
& \left(U_{1}^{s}(t)=0 \text { si } t<t s\right)
\end{aligned}
$$

Les hypothèses étant maintenant précisées, on se donne une loi de comportement pour le massif et pour le soutènement et on trace dans le diagramme $\mathrm{P}_{1}-\mathrm{U}_{1}$ (Fig. 3) :

\footnotetext{
- La donnée de $\mathrm{P}_{1}^{s}(\mathrm{X})$ résulte de la modélisation d'un problème essentiellement tridimensionnel. Par ailleurs cette fonction dépend de la loi de comportement de la roche que l'on étudie. Toutefois. cette dépendance est faible et l'erreur commise à ce niveau de second ordre.
} 


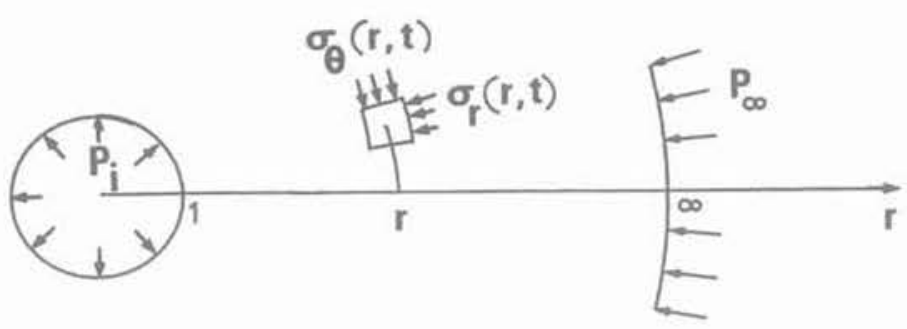

Fig. 1. - Géométrie et notations.

Fig. 1. - Geometry and notations.

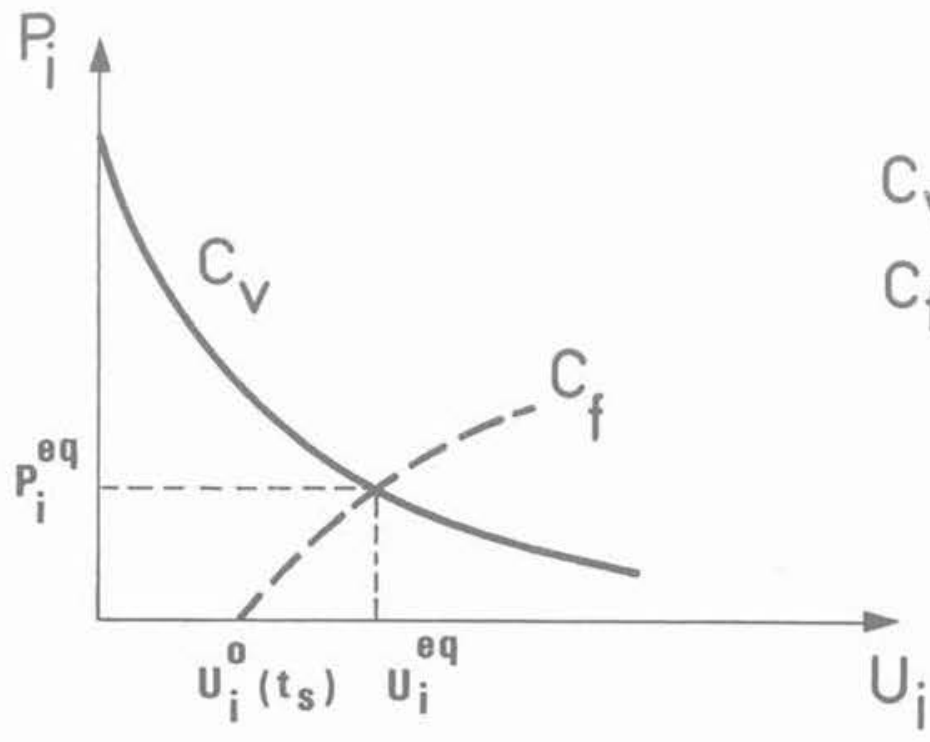

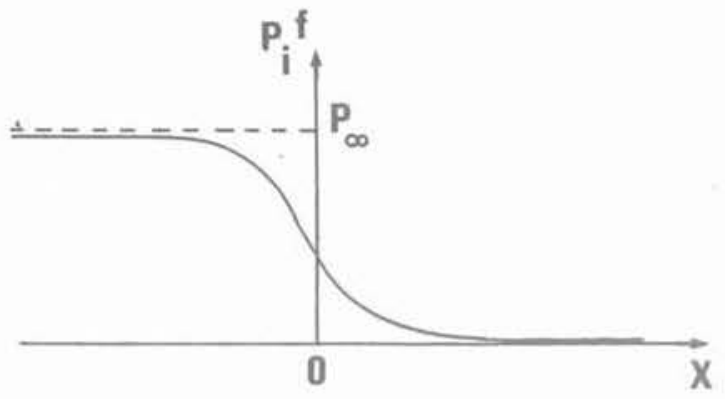

Fig. 2. - La pression fictive.

Fig. 2. - Fictitious pressure.

$C_{V}=$ courbe de convergence $C_{f}=$ courbe de confinement

Fig. 3. - Courbes de convergence et de confinement - Equilibre.

Fig. 3. - Convergence - confinement curves - Equilibrium.

- la courbe de convergence $\left(C_{v}\right)$ du massif qui ne prend en compte que la loi de comportement de la roche;

- la courbe de confinement $\left(\mathrm{C}_{\mathrm{f}}\right)$ du soutènement qui ne fait intervenir que la loi de comportement du soutènement.

L'intersection des deux courbes fournit le point d'équilibre du système, c'est-à-dire la convergence de la paroi $U_{1}^{e q}$ en fonction de la pression exercée par les terrains $\mathrm{P}$ eq

\subsection{Influence du «délai de pose»}

On constate sur la figure 3 que le deuxième paramètre fondamental précédemment cité, le délai de pose, a une importance prépondérante.

Il est en effet directement lié à la convergence $\mathrm{U}_{\mathrm{i}}^{\circ}$ (ts) avant pose dont la connaissance est essentielle pour l'estimation du point d'équilibre.

On trouvera sur la figure 4 l'illustration de 3 cas différents qui donnent le même point d'équilibre:

- Cas n ${ }^{\circ}$ 1: le soutènement est souple et peut donc être posé rapidement derrière le front ( $\mathrm{U}_{i}^{0}$ petit).
- Cas $n^{\circ} 2$ : le soutènement est raide: il faut donc laisser converger librement le massif avant de le poser ( $\mathrm{U}_{i}^{\circ}$ grand, donc délai de pose important).

- Cas n ${ }^{\circ} 3$ : le soutènement est à seuil de confinement: le comportement du soutènement n'est pas linéaire; il peut être posé rapidement.

\subsection{Application aux roches à effets différés}

La théorie classique, on l'a vu, ne donne qu'une seule courbe de convergence du massif; en particulier, la nature et la vitesse du chargement initial (creusement et pose) n'interviennent pas sur l'équilibre final. De ce point de vue, les 3 procédés décrits ci-dessus sont équivalents: pour un soutènement donné, l'équilibre ne dépend que du délai de mise en contact massifsoutènement.

La réalité est certainement plus complexe, surtout pour les matériaux plastiques de faible résistance mécanique et dont le comportement est sensible à l'effet de la contrainte moyenne d'une part (la plupart des roches ont une résistance qui augmente avec la pression moyenne) et du temps d'autre part.

Dans le cas $n^{\circ} 2$, par exemple, au cours de la première phase pendant laquelle il n'y a pas de soutè- 

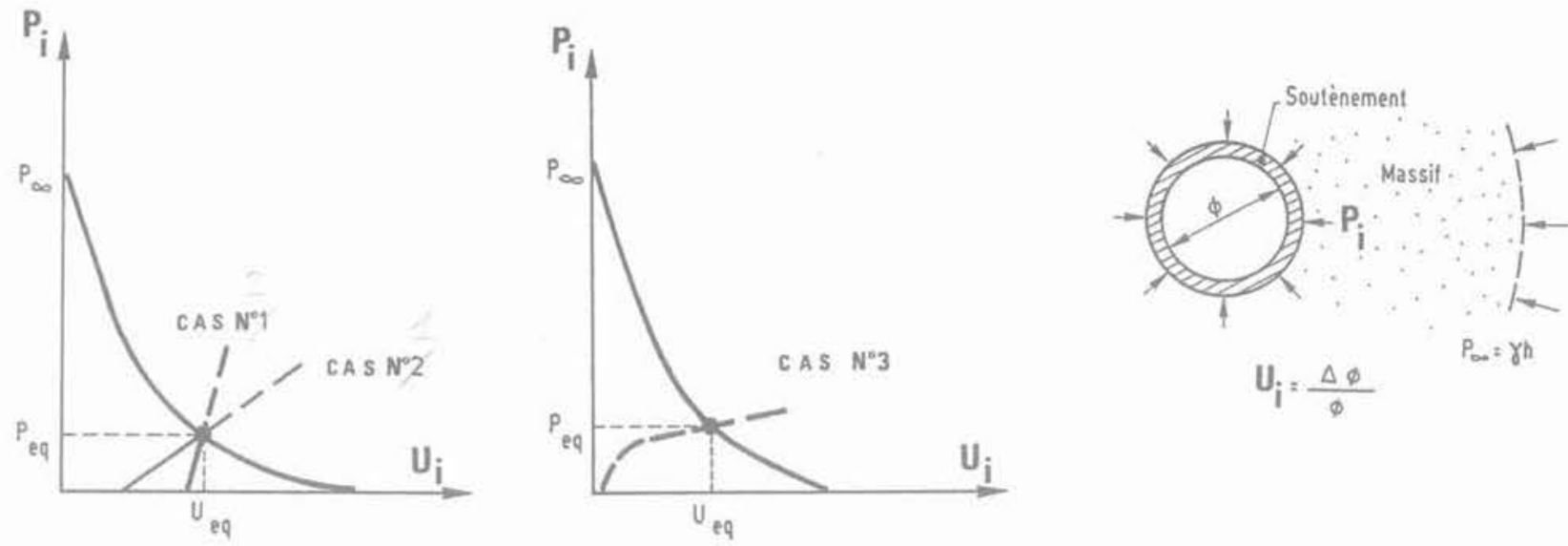

Fig. 4. - Influence de la courbe de confinement.

Fig. 4. - Influence of the confinement curve.

nement. la convergence n'est pas contrôlée. A la paroi, le déviateur des contraintes est très important et conduit à un développement important de la rupture: des fractures apparaissent et progressent à partir de la paroi.

Dans le cas $n^{\circ} 3$ au contraire, la phase pendant laquelle la paroi converge librement est réduite au minimum ( $\mathrm{U}_{\uparrow}^{\circ}$ faible). La majeure partie de la convergence se produit lorsque le soutènement est actif, c'est-à-dire sous confinement; la convergence est contrôlée, le déviateur réduit et la pression moyenne plus élevée.

On trouvera une illustration quantifiée de ce phénomène un peu plus loin.

Une deuxième limitation de la méthode pour les roches à effets différés est qu'elle ne donne que l'équilibre à long terme du système. Elle est inapte à donner le détail de l'évolution des convergences et pressions.

D'un point de vue théorique cette limitation n'est pas très importante, car le point d'équilibre à long terme est celui qui conduit aux sollicitations maximales dans le soutènement.

D'un point de vue pratique par contre, le problème essentiel est de déterminer les capacités de résistance mécanique et de déformabilité à long terme de la roche, de façon à pouvoir tracer la courbe de convergence à long terme. On conçoit aisément que cette étude du comportement à long terme est délicate; quelques éléments de cette étude sont présentés un peu plus loin.

Enfin, la troisième difficulté posée par les roches à effets différés est que la valeur de $U^{\circ}$. essentielle pour connaitre la pression de confinement, dépend, en plus du délai de pose, du premier paramètre fondamental: la vitesse de creusement et plus généralement des conditions de réalisation de louvrage.

\section{LOIS DE COMPORTEMENT OBTENTION DE LA COURBE DE CONVERGENCE A LONG TERME}

Les effets différés constatés sur un certain nombre d'ouvrages ont plusieurs causes possibles:
- le comportement de la roche est visqueux (cas du sel gemme, des marnes, des argiles,...);

- la roche est poreuse et peu perméable: les écoulements de l'eau interstitielle et la redistribution des contraintes effectives sous l'effet de l'évolution de la pression d'eau dans le massif se font progressivement.

\subsection{Référence aux essais de laboratoire}

De nombreux essais de laboratoire ont été développés pour étudier le comportement différé des roches.

L'essai de fluage, qui consiste à mesurer la déformation d'un échantillon de roche cylindrique soumis sur ses faces à des contraintes constantes, est l'un des plus connus (Fig. 5). En général, une étude de ce type conduit à la détermination des paramètres suivants:

- les caractéristiques de «résistance à long terme» du matériau, c'est-à-dire le déviateur $\mathrm{Q}$ - P lou plus généralement l'ensemble des couples, $P, Q$ ) en deçà duquel les effets différés s'estompent au bout d'un certain temps;

- les vitesses d'évolution des déformations.

Les essais sur tube épais, bien que la plupart du temps utilisés pour déterminer les caractéristiques de rupture à court terme, présentent l'avantage supplémentaire d'avoir une géométrie comparable à celle des tunnels et donc particulièrement bien adaptée à la théorie convergence confinement (Fig. 6). L'extrapolation des résultats de laboratoire au cas d'un ouvrage réel est facilitée, à condition bien sûr que l'effet d'échelle soit modéré.

Le laboratoire de Mécanique des Solides (ROUSSET, BAZARGAN, LENAIN, 1989, [14]) a développé récemment un appareillage et une procédure spécialement adaptés à l'obtention de la courbe de convergence à long terme pour les roches à effets différés.

Dans cet essai, la pression extérieure $\mathrm{P}_{e}$ rend compte de la pression lithostatique et la pression intérieure $P_{1}$ de la pression de confinement.

L'essai de fluage par palier, par exemple, consiste, après avoir placé l'échantillon sous conditions de con- 

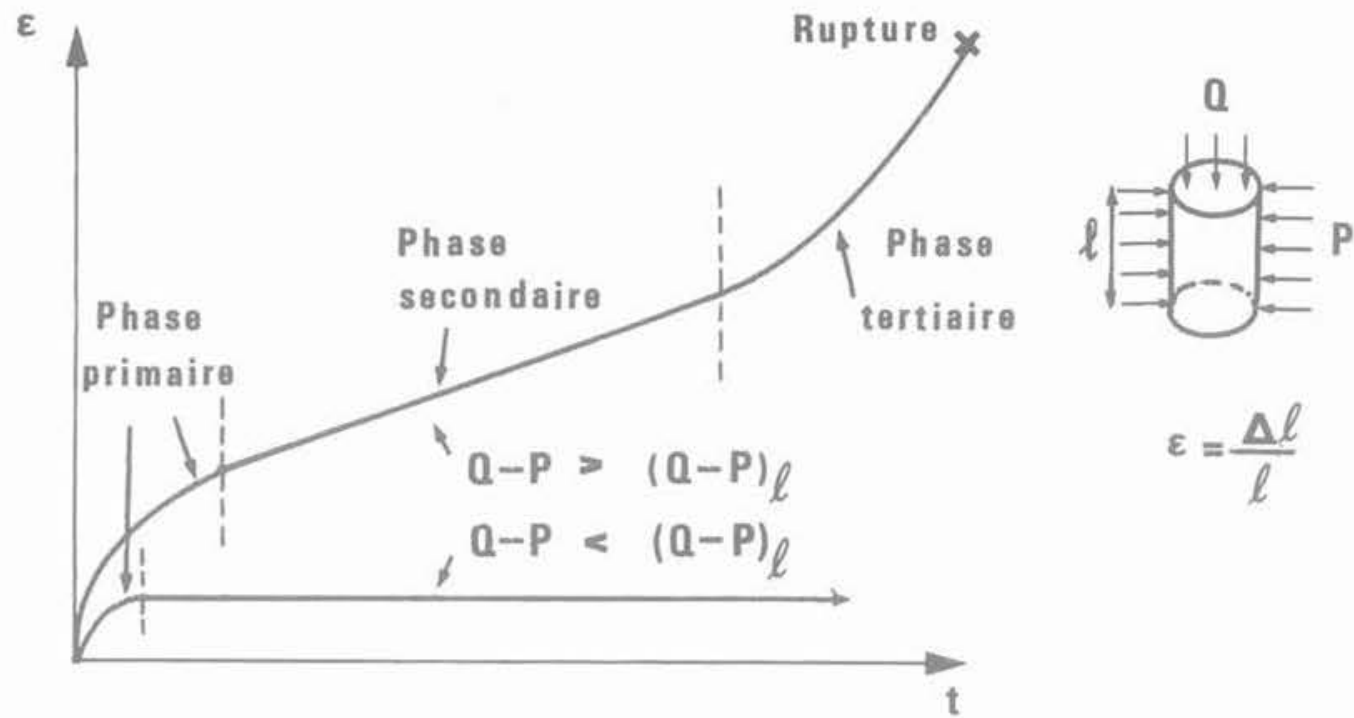

Fig. 5. - Essai de fluage.

Fig. 5. - Creep test
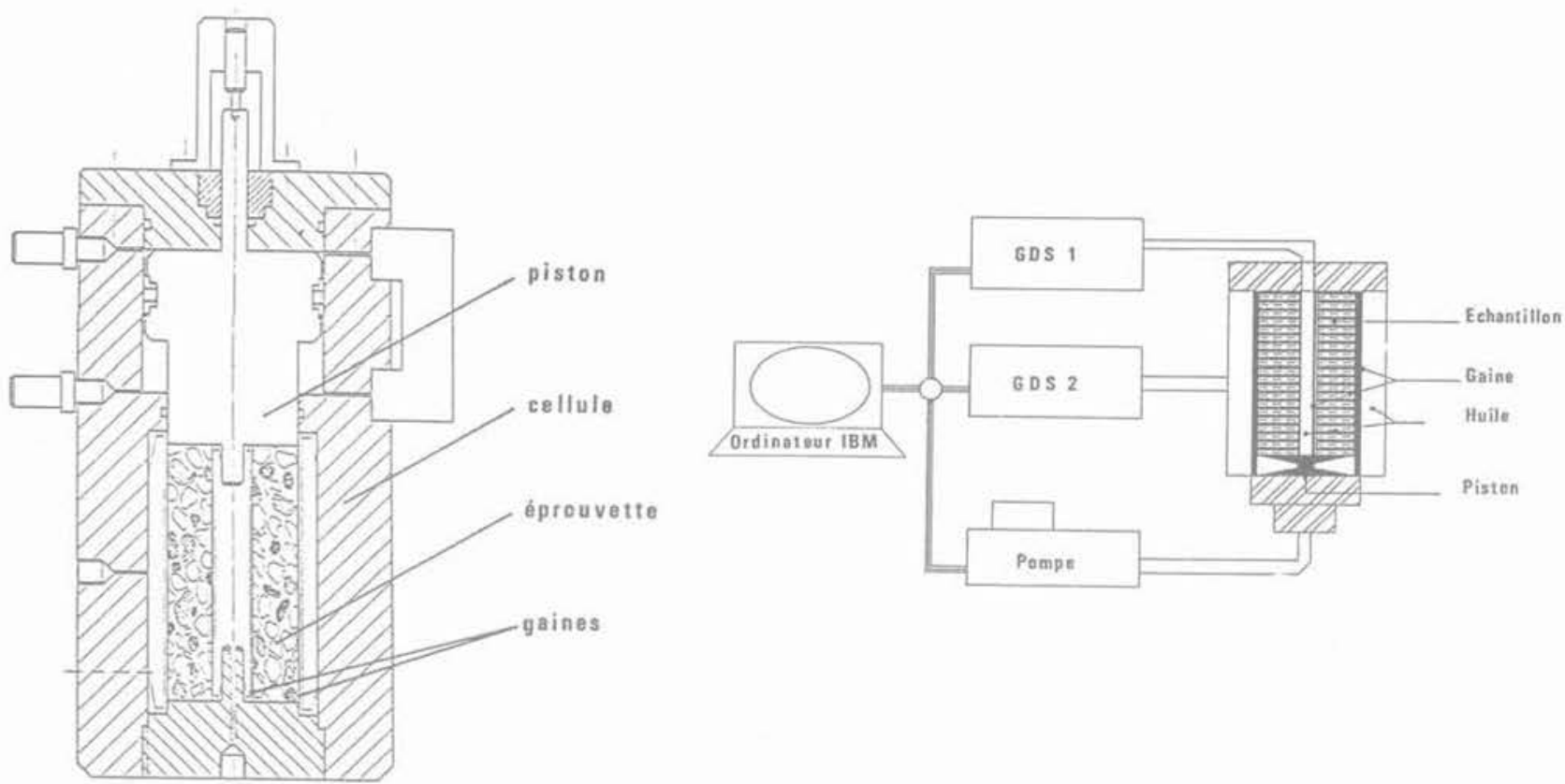

Fig. 6. - Essai sur tube épais.

Fig. 6. - Hollow cylinder test.

traintes isotropes (pression lithostatique), à créer un déviateur $\mathrm{P}_{e}-\mathrm{P}_{\mathrm{i}}$, par diminution de $\mathrm{P}_{1}$.

La convergence (variation du volume du trou) évolue et conduit:

- soit à la stabilisation, ce qui fournit un point sur la courbe de convergence à long terme et une indication sur la constante de temps des phénomènes: le processus est ensuite répété (changement de palier);

- soit à la rupture, ce qui donne une indication sur la valeur minimale du confinement que l'on peut espérer atteindre ainsi que sur le mode de rupture.

Quelques résultats expérimentaux sont détaillés plus loin (Fig. 14 et 15).

\subsection{Modèles rhéologiques}

L'étape suivante de la démarche consiste à proposer, à partir des résultats des essais, un modèle rhéologique qui rende compte des principaux aspects du comportement mécanique ainsi mis en évidence. La figure 7 illustre quelques représentations de ces modèles dans le cas unidimensionnel.

Un modèle complet consiste à associer divers éléments entre eux, le ressort (élasticité), le patin (plasticité) et l'amortisseur (viscosité). Lors du passage au cas tridimensionnel, des raffinements multiples sont proposés par de nombreux auteurs pour caler les modèles aux comportements expérimentaux. 


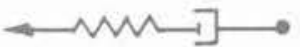

MAXWELL

(2) VISCOPLASTIQUES

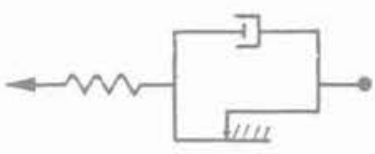

BINGHAM

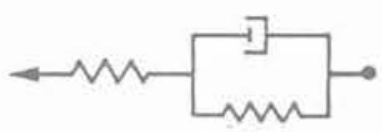

KELVIN - ST VENANT

Fig. 7. - Unidimensional representation of constitutive laws.

Dans le cas qui nous occupe on citera les trois raffinements les plus importants:

- la dilatance de la roche dès que la déformation dépasse un certain seuil;

- l'existence d'un angle de frottement, qui signifie que la "résistance» de la roche est d'autant plus élevée que la contrainte moyenne est grande;

- le radoucissement qui traduit la perte progressive de résistance mécanique de la roche dès que les déformations dépassent un certain seuil.

Pour le problème du tunnel, dès que la convergence de la paroi dépasse quelques pour cent, il est clair que les comportements irréversibles (plasticité) de la roche doivent être pris en compte.

Les modèles de type viscoplastiques semblent donc être les mieux adaptés (NGUYEN MINH DUC, 1986. [8]). La figure 8 illustre deux de ces modèles:

- le modèle de Bingham, qui est le plus simple, consiste à mettre en série un ressort (élasticité) et un ensemble patin-amortisseur montés en parallèle (viscoplasticité).

Si l'on choisit un seuil viscoplastique avec radoucissement on rend bien compte de l'effet des vitesses de chargement sur la "réponse» du matériau ainsi que sa rupture différée.

- le modèle viscoplastique avec rupture, (NGUYEN MINH D., ROUSSET G. 1987, [6]) consiste à rajouter au modèle précédent un élément plastique sans viscosité pour rendre compte de la rupture à court terme de la roche.

Il est utile pour déterminer l'influence des phases initiales (creusement et pose) sur le comportement à long terme du tunnel

\section{CALCUL DU TUNNEL-APPLICATION AU DIMENSIONNEMENT DES OUVRAGES}

\subsection{Démarche}

La dernière étape concerne le calcul qui doit servir au dimensionnement de l'ouvrage et à l'optimisation des paramètres de creusement.

La difficulté de cette étape ne réside pas dans le calcul lui-même mais plutôt dans la modélisation de la pression fictive, $\mathrm{P}$ f dont on a vu l'importance sur la réponse à court et long terme de l'ouvrage dans le cas des roches viscoplastiques.
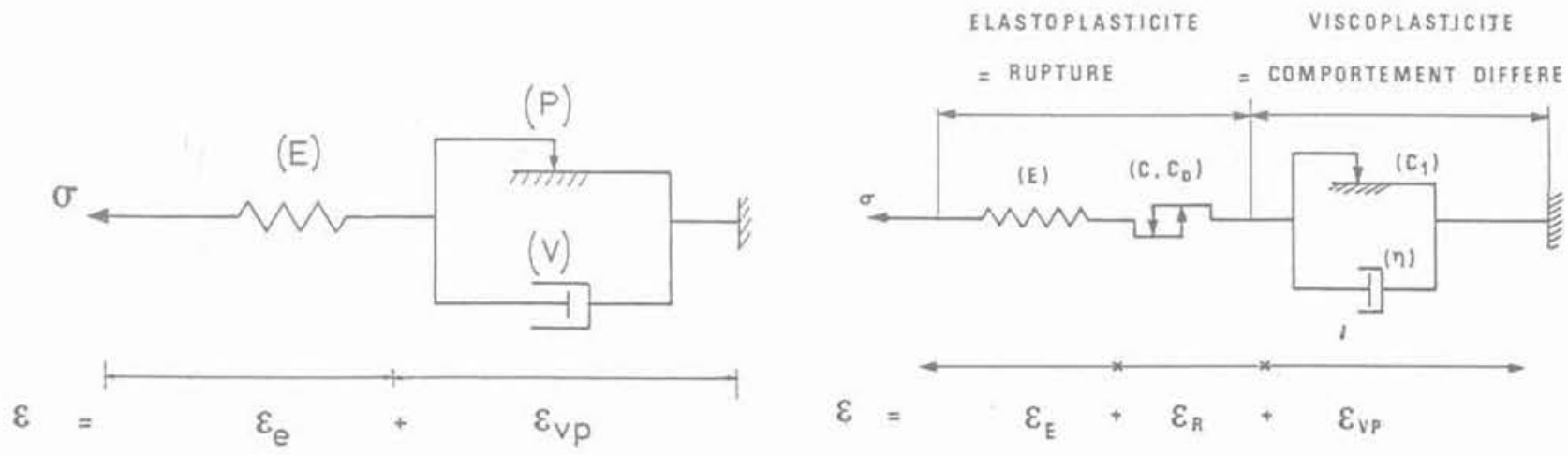

Fig. 8. - Modèles viscoplastiques.

Fig. 8. - Viscoplastic constitutive models. 
- Quelle forme de $\mathrm{P}_{1}^{f}$ fonction de la distance au front faut-il choisir?

- Quelle est la valeur de $\lambda$ (ou de $U_{1}^{\circ}$ ) qui correspond à la pose du soutènement?

Actuellement, la réponse à ces questions conserve un caractère empirique.

Du point de vue du calcul proprement dit, on montre qu'en général une zone viscoplastique de rayon y se développe à partir de la paroi (Fig. 9); dans cette zone, la vitesse de déformation viscoplastique est fonction du champ des contraintes et de la (ou des) variable d'écrouissage $\alpha$ (radoucissement). Elle s'écrit:

$$
\frac{\partial \epsilon^{v p}}{\partial t}=\frac{1}{\eta}<f_{v p}(\sigma, \alpha)>^{n} \frac{\partial g}{\partial \sigma}
$$

où $f_{v p}$ est le critère viscoplastique choisi. Pour le critère de Coulomb, $\mathrm{f}_{\mathrm{vp}}$ prend la forme suivante:

$f_{\mathrm{vp}}(\sigma, \alpha)=\sigma_{1}-\sigma_{3}+\left(\mathrm{K}_{\mathrm{p}}-1\right)\left(\sigma_{1}-\mathrm{H}(\alpha)\right)$

formule dans laquelle $\sigma_{1}, \sigma_{2}, \sigma_{3}$ sont les contraintes principales ordonnées, $K_{\mathrm{p}}$ le coefficient de poussée et $\mathrm{H}$ est relié à la cohésion.

$$
\mathrm{K}_{\mathrm{p}}=\operatorname{tg}^{2}\left[\frac{\pi}{4}+\frac{\phi}{2}\right] \quad \mathrm{H}=\mathrm{C} \operatorname{Cotg} \phi
$$

\subsection{Cas du comportement viscoplastique avec radoucissement}

La façon la plus simple de tenir compte du radoucissement est de considérer que la cohésion $\mathrm{C}$ du matériau diminue au fur et à mesure que la déformation viscoplastique augmente (Fig. 10). (BEREST P. et NGUYEN MINH D., 1979, [2]).
La forme de $\mathrm{P}_{\text {f }}$ et l'instant de pose du soutènement étant choisis, le calcul donne l'évolution de la convergence de la pression de soutènement et des zones viscoplastique ou de rupture. Un exemple numérique est présenté dans le paragraphe 5 .

\subsection{Cas du comportement viscoplastique avec rupture}

Dans le cas où l'on tient compte de la rupture du matériau en plus de sa viscoplasticité, l'influence de la vitesse du front pendant la phase de creusement et de la raideur du soutènement est déterminante comme on l'a déjà mentionné.

Les résultats portés sur la figure 11 illustrent ce phénomène. On a tracé, dans le cas simple où les critères viscoplastiques et de rupture sont des critères de Tresca (pas d'angle de frottement) la courbe $\mathrm{P}_{\infty}-\mathrm{P}_{1}$ en fonction du temps.

La modélisation du chargement est simple: pour la phase de creusement, on considère que la vitesse $A_{0}$ d'évolution de la convergence est constante $\left(A_{0}\right.$ est donc une mesure simplifiée de la vitesse d'avancement du front); dès que la convergence atteint une certaine valeur, un soutènement de grande raideur (infinie dans le calcul) est posé.

Le diagramme montre notamment que la pression de soutènement à l'équilibre est une fonction croissante de la vitesse initiale du front de taille: plus le creusement est rapide, plus la zone de rupture se développe et plus la pression de soutènement finale doit être élevée pour limiter la convergence à un seuil donné.

Ainsi la courbe de convergence à long terme du massif n'est plus unique comme le prévoyait la théorie classique convergence-confinement.
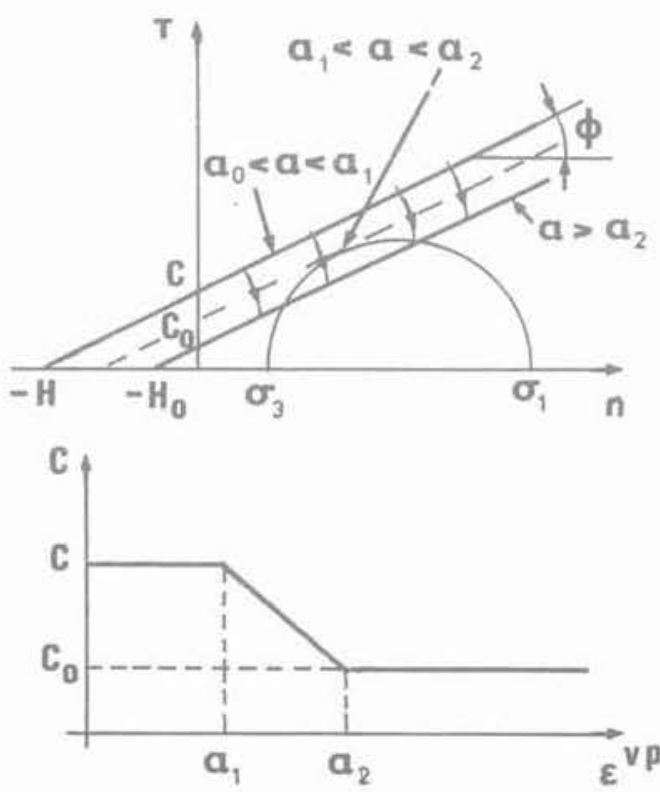

Fig. 9. - Développement de la viscoplasticité. Fig. 9. - Extension of viscoplastic zone.
Fig. 10. - Seuil viscoplastique de Coulomb avec écrouissage. Fig. 10. - Coulomb's viscoplastic yield limit with strain softening. 


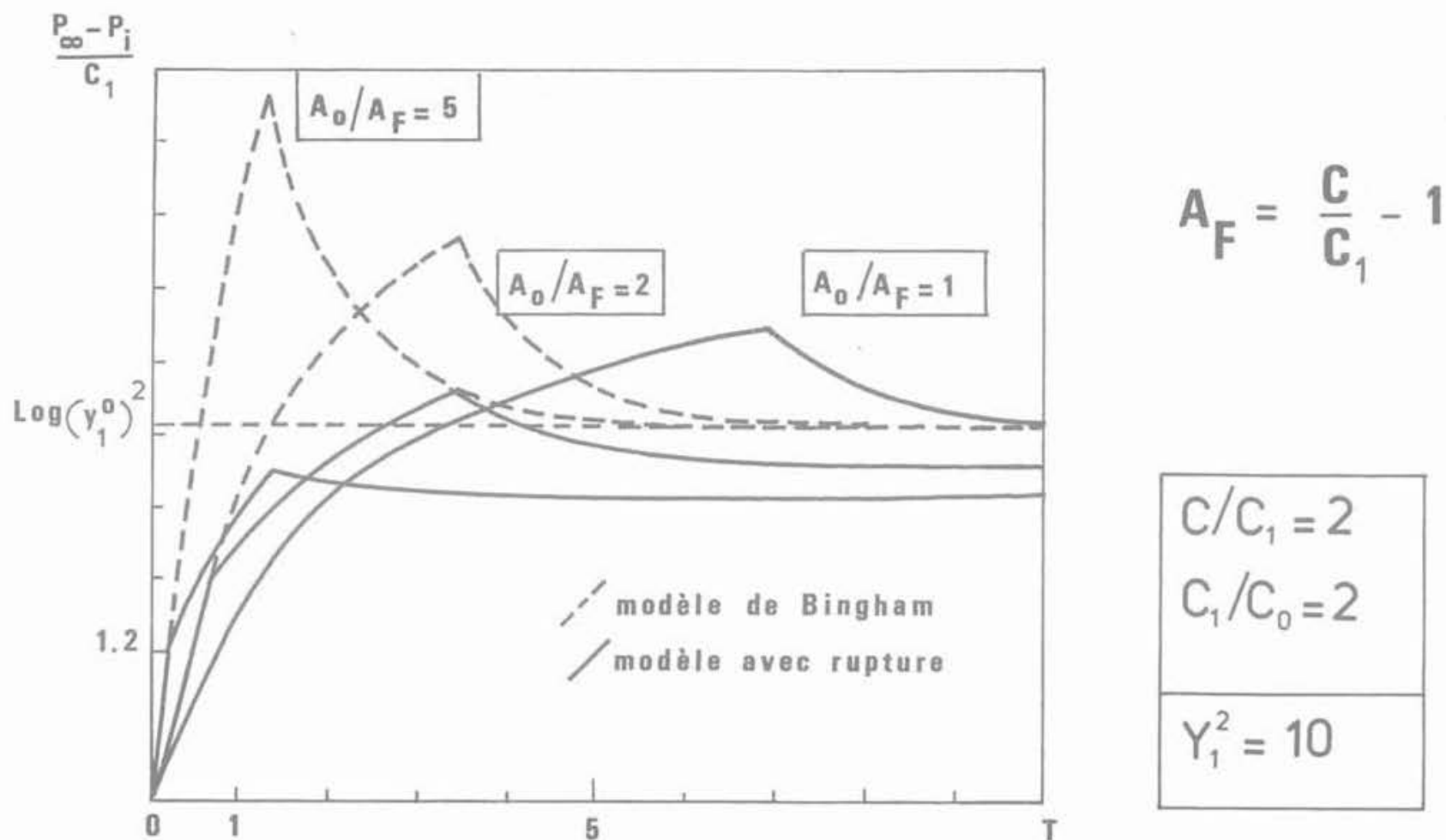

Fig. 11. - Pression de confinement en fonction du temps pour différentes vitesses de creusement. Fig. 11. - Continement pressure versus time for different tunnel advance rates.

La figure 12 montre les différences notables que l'on peut ainsi obtenir.

Pour une même convergence avant pose donnée ( $\mathrm{U}_{i}^{\circ}$ donné) si le creusement est lent, la rupture n'est pas atteinte et la courbe de convergence à long terme Co est unique. Le point d'équilibre est Mo. A l'inverse, si la vitesse de creusement est très rapide (c'està-dire si la constante de temps du creusement peut être considérée comme très petite vis-à-vis de la constante de temps de la viscosité $\eta / E$ ), le point d'équilibre $M_{\infty}$ est différent, la pression finale de soutène- ment étant plus élevée. $\mathrm{Ce}$ point $\mathrm{M}_{\infty}$ dépend en plus de la raideur du soutènement.

\section{APPLICATION DE LA DÉMARCHE À UN CAS CONCRET}

Dans la suite du texte nous allons nous intéresser à un cas concret, qui est celui de l'argile de Boom à Mol (Belgique).

Le Centre d'Etudes Nucléaires belge (CEN/SCK) possède des installations souterraines dans une couche
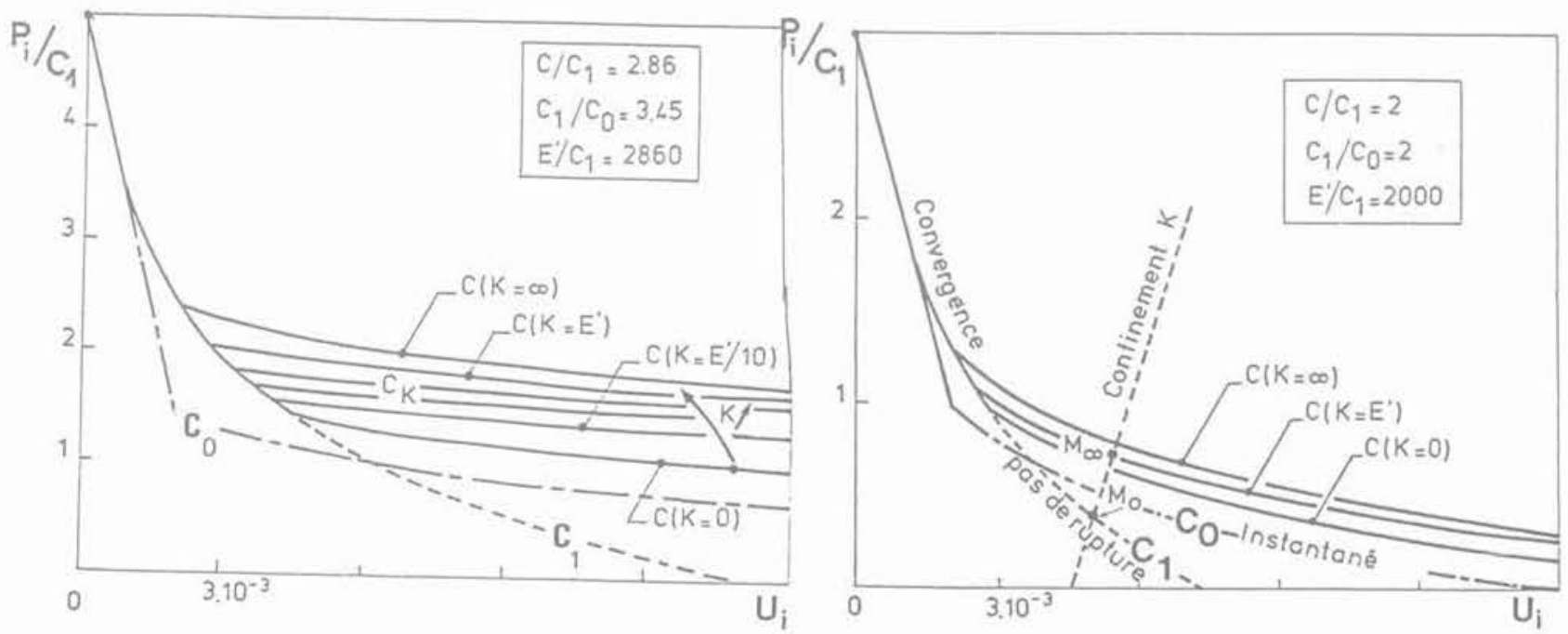

Fig. 12. - Courbes de convergence à long terme - Cas du comportement viscoplastique avec rupture. Fig 12. - Long terme convergence curve - Case of viscoplastic-faiture behavior 
d'argile homogène à 230 mètres de profondeur, qui constituent un laboratoire souterrain pour l'étude du stockage des déchets radioactifs.

L'argile de Boom est formée de $25 \%$ de matériaux grossiers et de $75 \%$ d'argile (dont $41 \%$ de smectite et $17 \%$ d'illite).

Elle est caractérisée par une teneur en eau de $23 \%$ et une densité moyenne de 2 ; il s'agit d'une argile "plastique» (limite de plasticité $=25$ à $27 \%$; indice de plasticité $=40$ à $48 \%$ )

\subsection{Comportement mécanique à court terme}

La figure 13 illustre les résultats obtenus lors d'un essai triaxial non drainé, sans ou avec cycles. On retiendra notamment:

- l'existence d'une phase de «plasticité parfaite» étendue ( $\epsilon$ de 2 à $5 \%$ );

- un radoucissement important pour des fortes déformations $(\epsilon>5 \%)$;

- l'absence de partie élastique, mise en évidence par les essais cycliques;

- un écrouissage très important;

- l'ensemble des essais effectués avec différents confinements met en évidence l'existence d'un angle de frottement interne non drainé peu élevé, $\phi=4^{\circ}$ et une valeur de la cohésion à court terme de $1,3 \mathrm{MPa}$.

\subsection{Comportement mécanique à long terme}

1000 heures de fluage environ ont été nécessaires pour caractériser finement le comportement différé. Les résultats obtenus sont qualitativement indiqués dans le tableau 1.
Tableau 1. - Résultats des essais de fluage. Table 1. - Creep tests results.

\begin{tabular}{|c|c|c|}
\hline $\begin{array}{l}\text { Déviateurs } \\
(\mathrm{Q}-\mathrm{P})(\mathrm{MPa})\end{array}$ & $\begin{array}{l}\text { Rapports } \\
(Q-P) / \\
(Q-P)_{M}\end{array}$ & Commentaires \\
\hline$<1,5$ & & Peu ou pas de fluage \\
\hline 1,5 ou 1,75 & $43 \%$ ou $50 \%$ & $\begin{array}{l}\text { Fluage primaire (déformation } \\
\text { de l'ordre de } 1 \% \text { ) } \\
\text { Pas de fluage secondaire }\end{array}$ \\
\hline 2 ou 2,25 & $57 \%$ ou $64 \%$ & $\begin{array}{l}\text { Fluage secondaire dans la plu- } \\
\text { part des cas } \\
\text { Déformations modérées }\end{array}$ \\
\hline 2,5 & $71 \%$ & $\begin{array}{l}\text { Fluage secondaire à vitesse } \\
\text { élevée, suivi parfois d'un flu- } \\
\text { age tertiaire ou de la rupture }\end{array}$ \\
\hline 3 & $86 \%$ & $\begin{array}{l}\text { Fluage secondaire à vitesse } \\
\text { très élevée ou rupture }\end{array}$ \\
\hline$>3$ & & Rupture instantanée \\
\hline
\end{tabular}

On notera en particulier que pour le confinement $P$ donné de $5 \mathrm{MPa}$ la «résistance à long terme» de la roche n'est égale qu'à $43 \%$ de sa résistance à court terme.

Les résultats des essais de fluage par palier sur tube épais illustrent d'une manière complémentaire ce comportement.

La figure 14 donne un exemple de résultats fournis par ce type d'essai.

Le chargement (courbe 14-a) consiste à diminuer par palier la pression intérieure à partir de la valeur de $5 \mathrm{MPa}$ (pression lithostatique à $250 \mathrm{~m}$ de profondeur). La réponse du système (courbe 14-b) donne la convergence en fonction du temps. Dans le diagramme convergence-confinement, il suffit de joindre les points
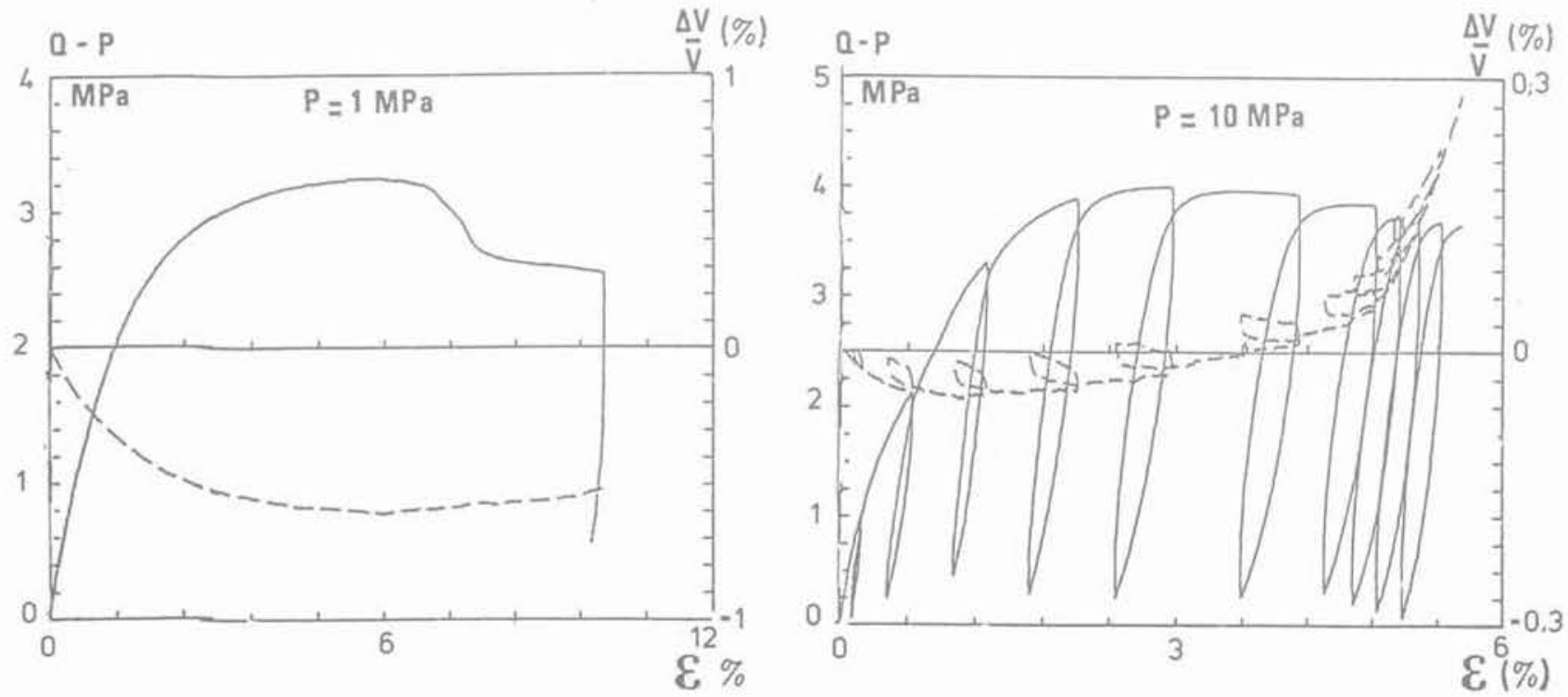

Fig. 13. - Essais triaxiaux sur l'argile de Boom.

Fig. 13. - Triaxial tests on Boom clay. 


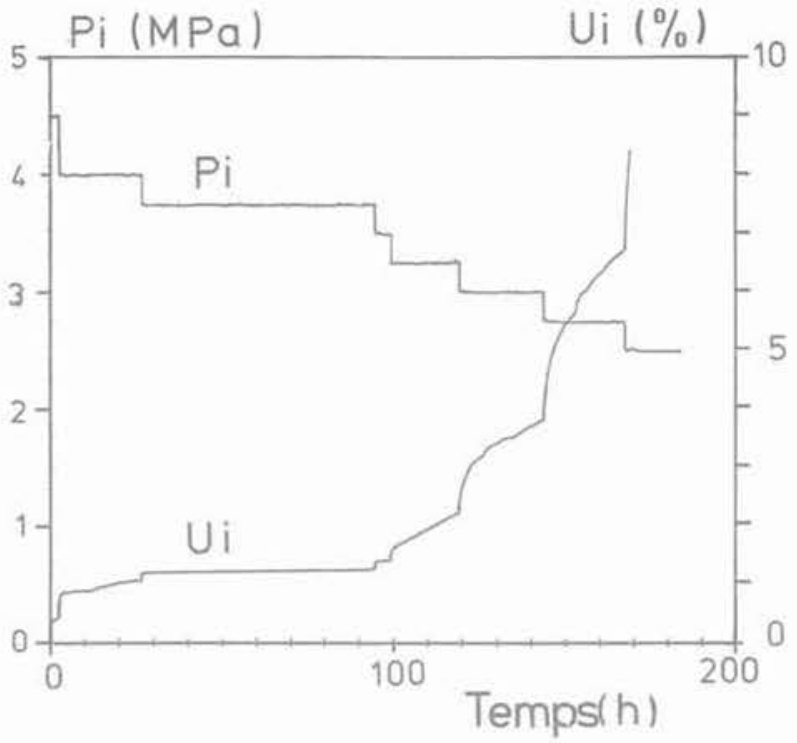

Fig. 14. - Essai de fluage par palier sur tube épais. Fig. 14. - Creep test on hollow cylinder.

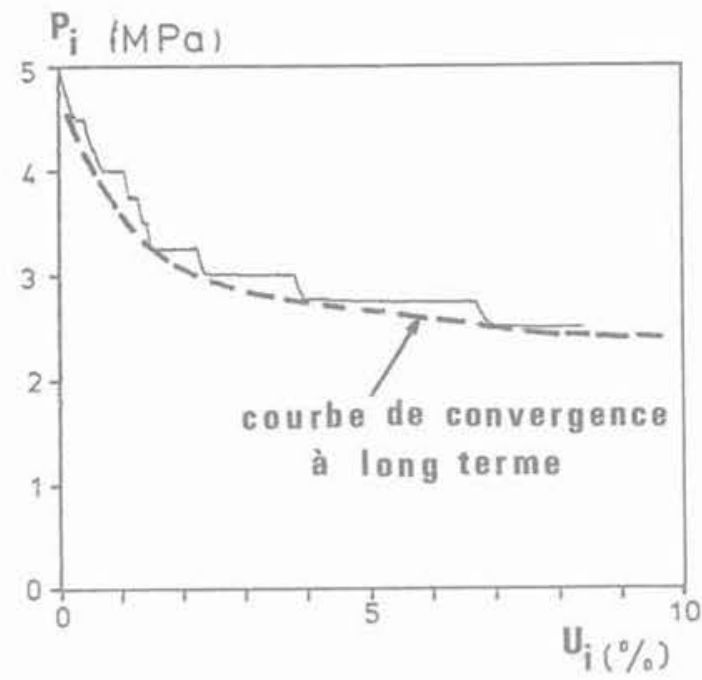

Fig. 15-a. qui correspondent à la stabilisation de chaque palier pour obtenir la courbe de convergence à long terme du matériau (Fig. 15-a).

Plusieurs essais de ce type ont été réalisés; la dispersion expérimentale est faible, comme on peut le constater sur la figure 15-b. On peut noter à nouveau la différence importante entre les courbes de convergence à court et long terme.

L'ensemble de ces essais nous permet de proposer un modèle de comportement précis et de caler les paramètres du modèle. On trouvera le détail de ce calage dans (G. ROUSSET, 1988, [13]). Ce modèle est illustré sur la figure 16 . Il comprend 10 paramètres:

- 5 pour décrire le seuil viscoplastique $\left(\epsilon_{0}, \epsilon_{1}, \epsilon_{2}\right.$, $\mathrm{C}$ et $\left.\mathrm{C}_{0}\right)$;

-2 pour la viscosité ( $\eta$ et $n$ );

- 2 pour l'élasticité ( $\mathrm{E}$ et $v$ ):

- langle de frottement $\phi$.

Il est caractérisé par un écrouissage très important et une forte non linéarité du comportement visqueux.

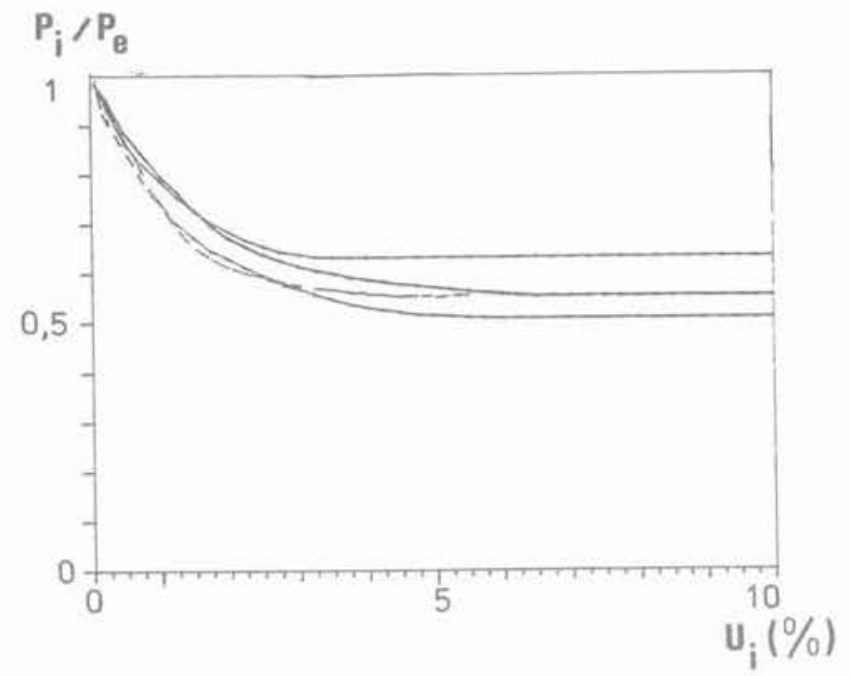

Fig. 15-b.

Fig. 15. - Courbes de convergence expérimentales.

Fig. 15. - Experimental convergence curve.
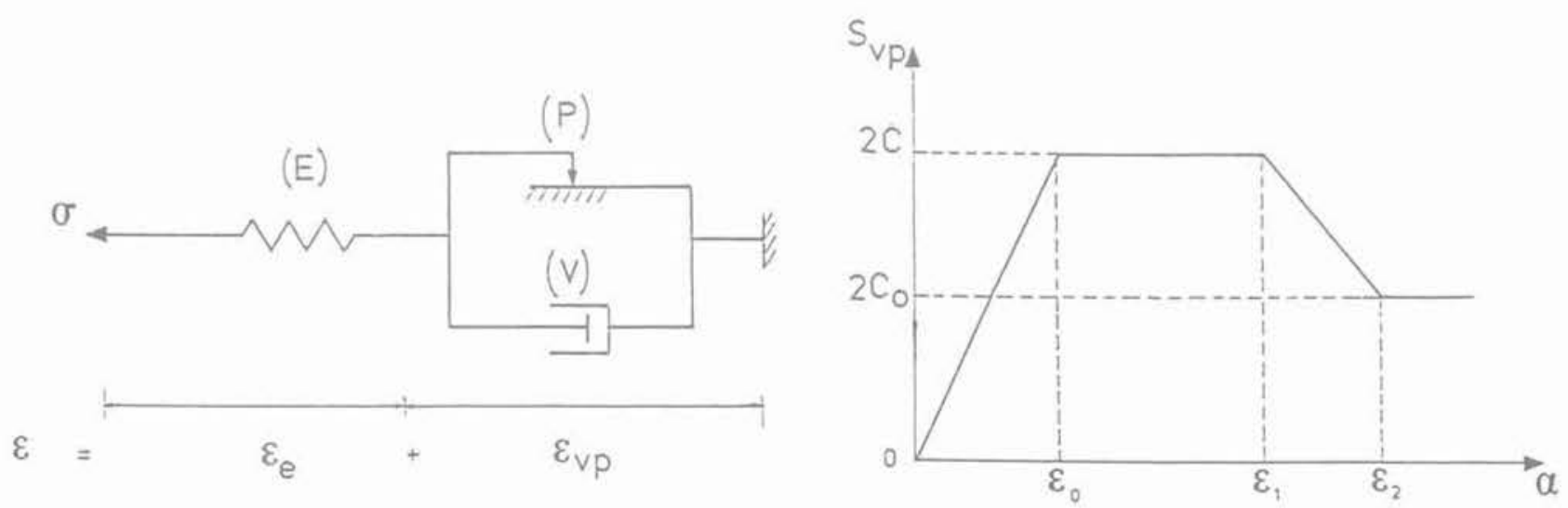

Fig. 16. - Modèle de comportement pour l'argile de Boom.

Fig. 16. - Constitutive model for Boom clay. 


\section{ESSAIS IN SITU - (RAMEAUX D'ESSAI ET MODĖLES RÉDUITS)}

L'étude sur échantillon en laboratoire, aussi complète soit-elle, ne remplace pas la mesure in situ. Deux raisons essentielles peuvent conduire à des différences appréciables entre les 2 approches; l'effet d'échelle et la méconnaissance du champ de contrainte lithostatique.

Si limportance du projet le permet, il sera très utile de réaliser un rameau d'essai ou des modèles réduits.

On présente dans ce paragraphe deux exemples de ce type d'essai réalisés à Mol.

Ces travaux font partie du programme d'étude du comportement mécanique des argiles profondes piloté par l'Agence Nationale pour la gestion des Déchets Radioactifs (CEA/ANDRA). Ils ont été financés en partie par la Commission des Communautés Européennes (CCE).

\subsection{Rameau d'essai}

L'ouvrage est une galerie de petites dimensions (diamètre utile $1,4 \mathrm{~m}$; longueur $7 \mathrm{~m}$ ) revêtue de claveaux de béton de $30 \mathrm{~cm}$ d'épaisseur (point 1 sur la figure 17).

L'ensemble de l'instrumentation mise en cuvre (cellule Glotzl, cellules de charge entre claveaux, convergence à fil d'Invar) permet de mesurer l'évolution de la pression de soutènement et de la convergence.

Le résultat le plus intéressant concerne les déplace. ments dans le massif. La configuration particulière des installations nous a en effet permis de placer un extensomètre dans le massif avant le creusement de la galerie. L'appareil donne les déplacements radiaux de cinq point différents du massif en fonction du temps et en particulier pendant la phase de creusement, (MANFROY et al., 1985, [7]).

On a donc ainsi une estimation de la convergence du massif avant la pose du soutènement (valeur de $\mathrm{U}^{\circ}$ ), c'est-à-dire à partir de l'état initial, ce qui est excep. tionnel en géotechnique.

La figure 18 donne les déplacements en fonction du temps; on remarquera notamment que l'excellente précision de la mesure permet de déceler des mouvements quatre années après la réalisation de l'ouvrage et, au début, met en évidence de façon tout à fait claire l'influence des phases de creusement sur les déplacements.

Enfin, on notera que les déplacements dus au creusement ne représentent qu'une faible partie des déplacements totaux. Les déplacements se produisant pendant les phases d'arrêt de chantier ou après la pose du soutènement sont largement les plus importants.

Si l'on porte ces résultats dans un diagramme LoguLogr (Fig. 19), on constate qu'à chaque instant les résultats relatifs aux cinq points de mesure sont alignés. La dépendance u(r) s'écrit donc:

$$
u(r)=\frac{U_{1}(t)}{r^{1,3}}
$$

La puissance de r étant supérieure à 1, on en déduit qu'il y a dilatance du matériau. Par extrapolation, on trouve la convergence de la paroi en fonction du

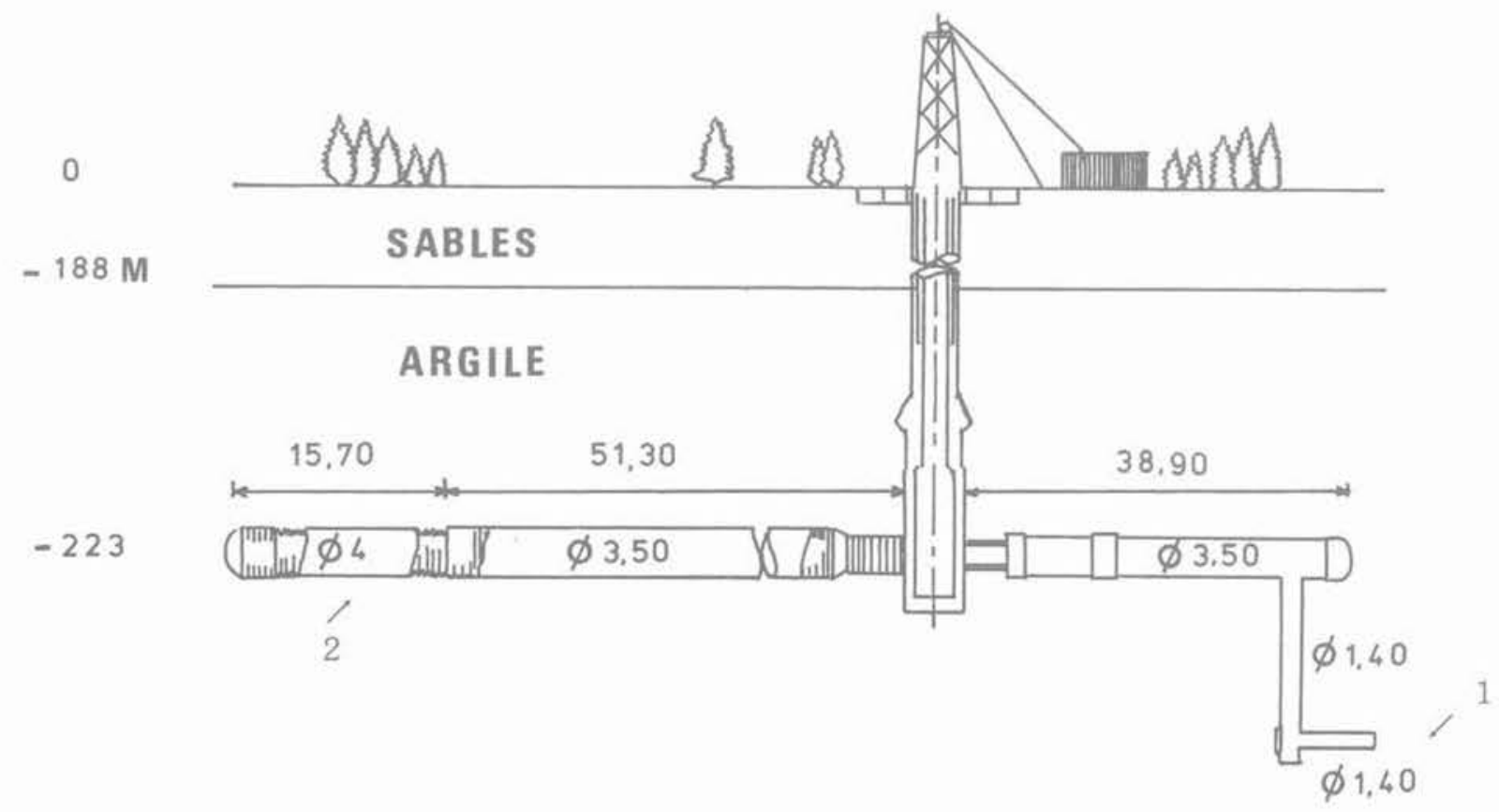

Fig. 17. - Installations souterraines de Mol.

Fig. 17. - Experimental facility at Mol. 

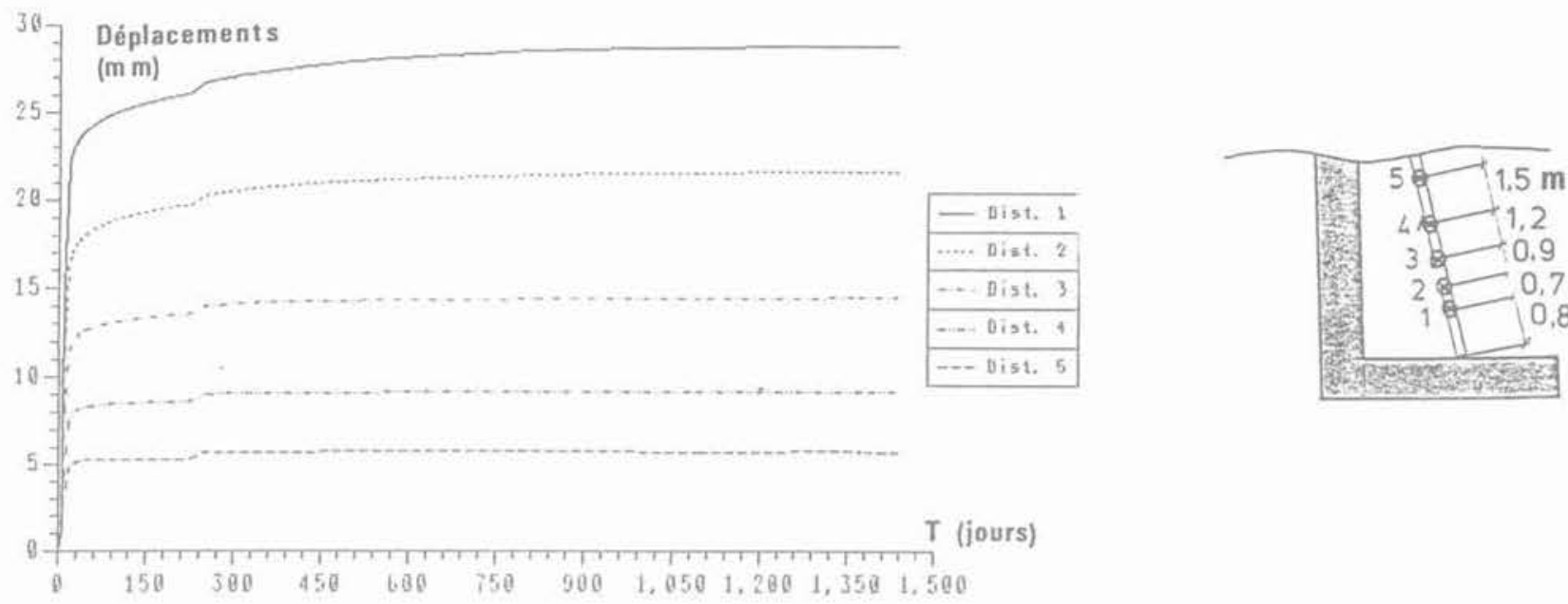

Fig. 18. - Mesures extensométriques.

Fig. 18. - Extensometric measurements
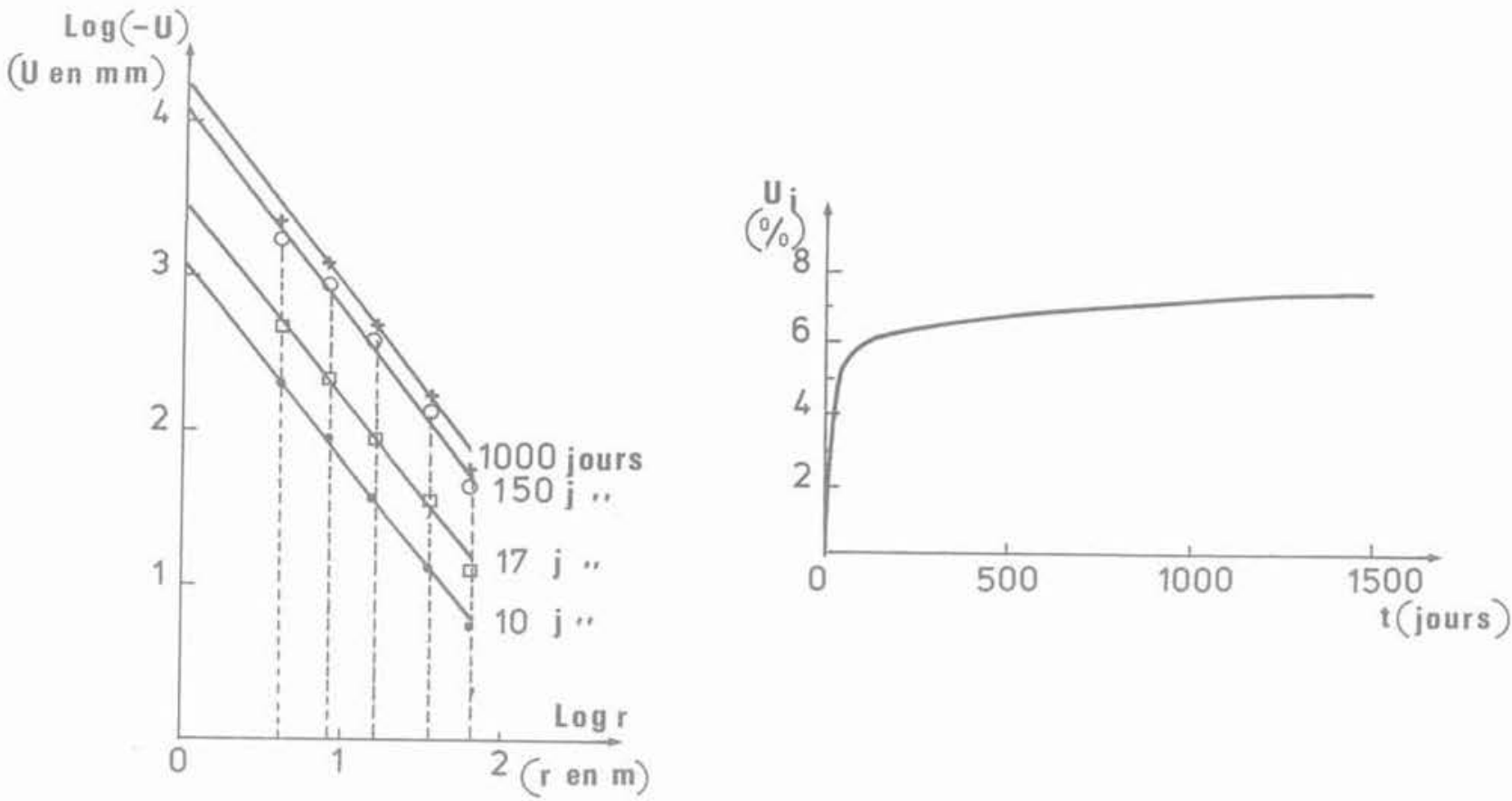

Fig. 19. - Déplacements en fonction de la distance à l'axe - Extrapolation à la convergence.

Fig. 19. - Displacements versus radius - Convergence.

temps qui atteint plus de $7 \%$ quatre années après la fin du creusement. Comme la convergence du soutènement n'excède pas $1 \%$, on en déduit que $85 \%$ de la convergence se produit avant que le soutènement ne soit réellement actif.

\subsection{Essai à petite échelle}

L'exemple présenté ci-dessous est destiné à étudier in situ à partir de forages de petits diamètres (entre 10 et $15 \mathrm{~cm}$ ), le comportement différé de la roche dans des conditions de chargement représentatives de celles du tunnel.

Il s'agit de l'essai de fluage par palier au dilatomètre dont le principe (ROUSSET, 1986, [12]) consiste à mesurer l'évolution globale de la convergence des parois du forage lorsqu'on impose une pression $\mathrm{P}$ constante par palier (mesure du volume $\mathrm{V}$ de la sonde et mesures de diamètre). Il s'agit donc de la mêrne procédure que celle utilisée lors des essais de fluage sur tube épais.

Les résultats (partiels) sont illustrés sur la figure 20 et montrent une fois encore l'importance des phénomènes différés. Dès à présent, 3 points expérimentaux d'équilibre peuvent être portés sur la courbe de convergence à long terme du massif:

$$
\begin{aligned}
& \left(P_{1}=3,2 \mathrm{MPa} U_{i}=0,9 \%\right), \\
& \left(P_{1}=2,6 \mathrm{MPa} U_{i}=1,7 \%\right), \\
& \left(P_{1}=1,9 \mathrm{MPa} U_{1}=6 \%\right) .
\end{aligned}
$$

\footnotetext{
- L'essai du fluage in situ au dilatomètre est réalisé en commun avec le BRGM pour le compte de l'ANDRA. II est financé en partie par la CCE.
} 


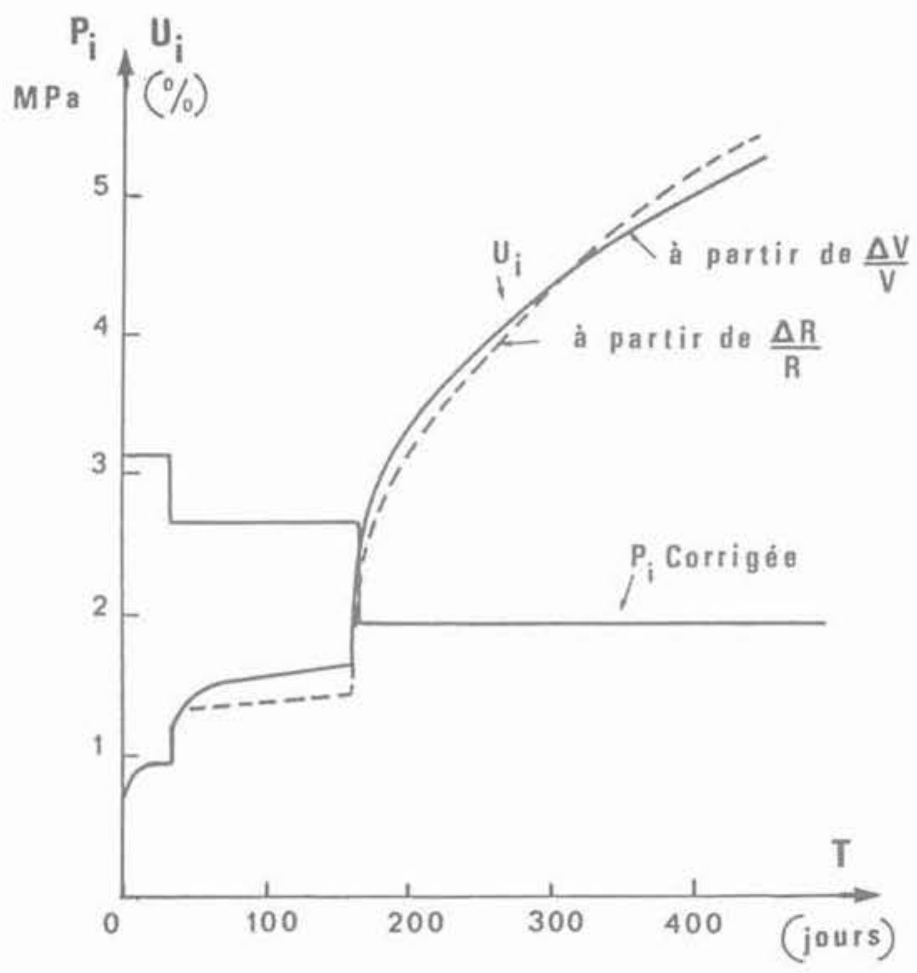

Fig. 20. - Essai de fluage par palier au dilatomètre.

Fig. 20. - In situ dilatometric creep test.

\section{L'ESSAI DE SOUTÈNEMENT À CONVERGENCE CONTRÔLÉE}

\subsection{Principe}

Afin de mettre à profit les capacités de résistance mécanique à long terme de la roche mises en évidence par l'ensemble des essais précédents, nous avons proposé à l'ANDRA et à la CCE de réaliser un ouvrage avec dimensionnement "optimisé » spécialement adapté aux roches viscoplastiques.

L'optimisation concerne le critère économique (soutènement léger et facile à mettre en ceuvre) et le critère sûreté (conserver l'intégrité du massif en limitant le développement de la fracturation).

Le soutènement à joints coulissants qui a été choisi permet de concilier en effet les deux objectifs essentiels décrits plus haut:

- il favorise la convergence grâce au coulissement et permet donc de limiter la pression de confinement, c'est-à-dire d'optimiser le dimensionnement du soutènement;

- il permet de limiter la convergence sans soutène. ment puisqu'il peut être posé immédiatement derrière le front de taille et que la vitesse d'avancement est élevée ( $\mathrm{U}_{\mathrm{i}}^{\circ}$ faible). Le développement de la rupture est ainsi réduit et la convergence se produit sous confinement: elle est contrôlée.

\subsection{Description de l'ouvrage}

L'ouvrage est constitué d'une galerie circulaire de $4 \mathrm{~m}$ de diamètre intérieur, revêtue de cintres coulissants de type TH 44/58 (Fig. 21).
Chaque anneau est formé de 4 éléments assemblés au moyen d'étriers spéciaux. Il est posé immédiatement derrière le front de taille (de façon à limiter les mouvements incontrôlés) et il est rendu solidaire de la paroi grâce à un colmatage efficace de l'espace annulaire (gunitage).

Dès que la poussée du massif devient suffisante, le frottement entre chaque élément dû au serrage des étriers n'est pas suffisant et le coulissement se déclenche.

Le seuil initial de coulissement $\mathrm{P}_{\text {f }}$ est de $1 \mathrm{MPa}$ environ (soit 4 ou 5 fois moins que la pression lithostatique).

Dès que le coulissement se produit la pression de confinement chute comme le prévoit la théorie convergence confinement. Elle augmente ensuite lentement, grâce au comportement différé du massif. Le processus se répète ensuite.

La courbe de confinement de ce soutènement est donc fortement non linéaire (cas $n^{\circ} 3$ de la figure 4).

\subsection{Instrumentation}

L'instrumentation très variée mise en œuvre par SIMECSOL permet de remonter de façon extrêmement précise aux efforts et déformations dans le soutènement. Elle est constituée des éléments suivants (Fig. 22):

- coulissement (mesure directe);

- convergence (fil d'Invar);

- pression totale (cellule Glotzl);

- extensométrie sur cintre (Kovari). 


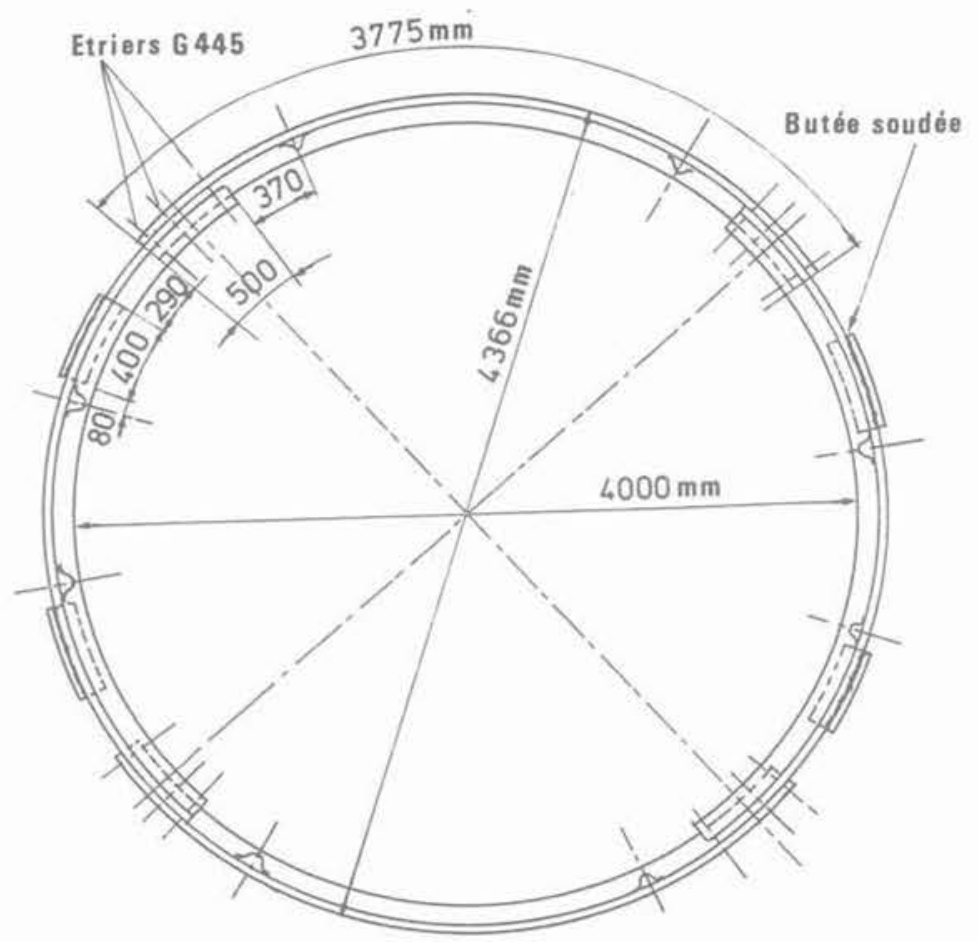

Fig. 21. - Cintre métallique.

Fig. 21, - Metallic rib.

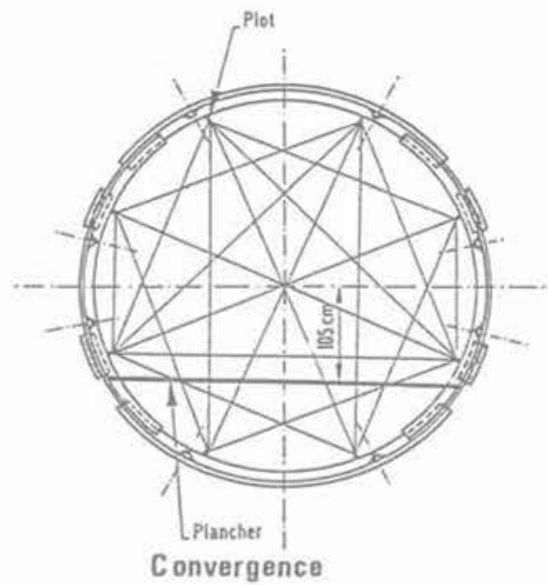

Fig. 22. - Instrumentation.

La dernière mesure est particulièrement intéressante car elle permet grâce à une combinaison des déformations mesurées à l'intrados, extrados et fibre neutre du cintre de calculer les efforts globaux dans le cintre (effet normal et moment fléchissant) et donc, après interprétation, les efforts de poussée du massif (confinement).

\subsection{Résultats}

(BUBLITZ D., 1988, [3], [15] et [16])

Coulissement (ou convergence) et pression totale moyens sont donnés en fonction du temps sur la figure 23. Les résultats sont conformes aux prévisions: après 15 mois de mesure, la convergence moyenne est de l'ordre de $2,2 \%$ pour un confinement de lordre de 1,5 MPa (estimé à partir des mesures d'extensométrie sur cintres). Les effets différés sont très marqués, plusieurs années seront nécessaires avant d'obtenir la stabilisation des phénomènes.

On notera également que le confinement estimé à partir des mesures d'extensométrie sur cintre est de 30 à $40 \%$ supérieur à celui mesuré directement au moyen des cellules Glotzl.

Ce résultat est conforme à ce que l'on observe généralement (les mesures directes de pression sont le plus souvent sous-estimées).

Les mêmes résultats portés dans le diagramme convergence-confinement donnent la courbe expérimentale de confinement de ce soutènement $\mathrm{C}_{f}$ (Fig. 24). Sa forme (de type élastoplastique avec léger 

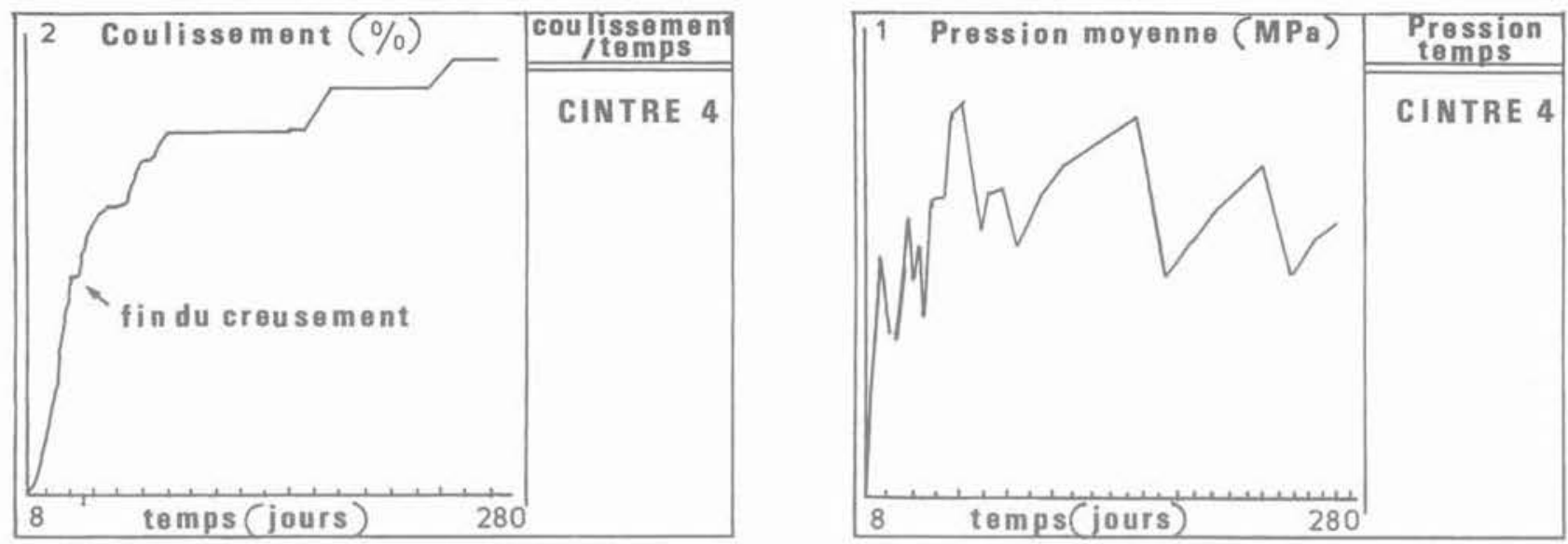

Fig. 23. - Convergence et confinement en fonction du temps.

Fig. 23. - Convergence and lining pressure versus time.

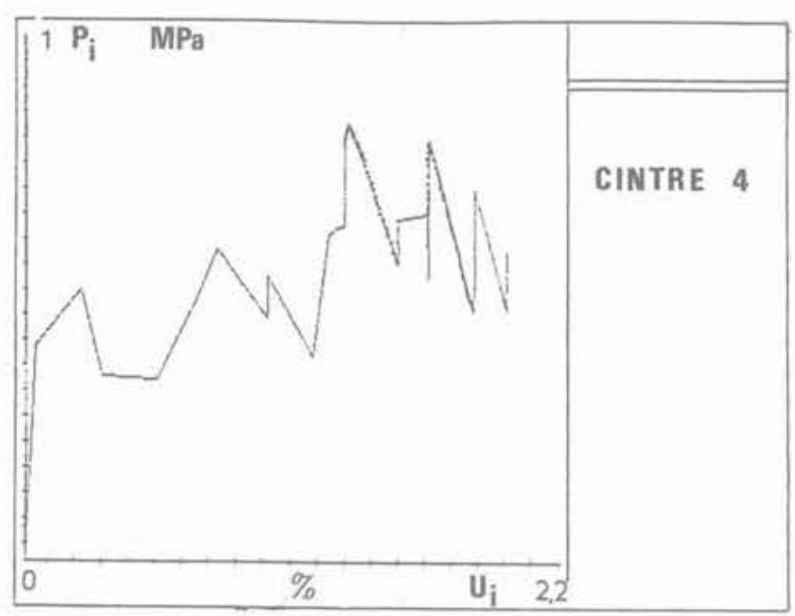

Fig. 24. - Courbes expérimentales de confinement $C_{f}$. Fig. 24. - Experimental confinement curves $C_{f}$.

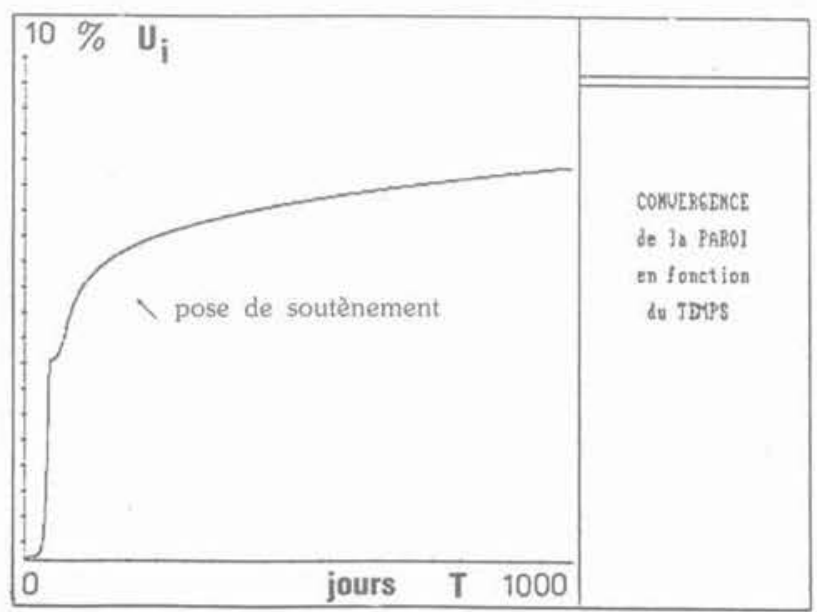

écrouissage moyen positif) est bien conforme à ce que l'on attendait.

La confrontation des résultats expérimentaux avec les résultats issus du calcul (avec la loi de comportement décrite ci-dessus) donne entièrement satisfaction (Fig. 25)

\section{CONCLUSION}

On retiendra de cette étude une démarche intéressante pour l'application de la méthode de convergence-confinement au dimensionnement des ouvrages souterrains dans des roches à comportement différé.

En particulier, on a montré comment à partir d'essais de laboratoire, il était possible d'estimer les caractéristiques du comportement différé ainsi que les capacités de résistance mécanique à long terme de ces roches.

La bibliothèque des lois de comportement permet de caler un modèle qui rende compte des principales caractéristiques du comportement mécanique.

Fig. 25. - Résultats de la modélisation.

Fig. 25. - Modelling resuits. 
L'extrapolation au comportement in situ reste évidemment le problème le plus délicat, surtout pour les roches où l'effet d'échelle est important.

Si l'importance du projet le permet, il est très utile de procéder à des expériences in situ à échelle réduite (rameau d'essai ou essais en forage). Les quelques exemples décrits dans ce texte montrent qu'il est possible d'extrapoler avec plus de certitude les résultats ainsi obtenus à des ouvrages réels.

Enfin, l'auscultation de l'ouvrage, très utile pour vérifier son dimensionnement est essentielle.

Le problème est là aussi difficile. Il vaut mieux privilégier les mesures globales dans le soutènement (contraintes, déformations, convergence) plutôt que les mesures ponctuelles de pression dont l'interprétation est en général malaisée.

\section{BIBLIOGRAPHIE}

[1] AFTES, «Stabilité des tunnels par la méthode convergence-confinement», Journées d'étude, Paris, 26 octobre 1978.

[2] BEREST P., NGUYEN MINH D. (1979), «Etude en grande déformation de la contraction et de l'expansion de cylindres creux de révolution élastoplastiques avec radoucissement», Journal de Mécanique Appliquée, vol. 3, nº4.

[3] BUBLITZ D. (1988), "Essai de soutènement à convergence contrôlée - Interprétation des mesures», Document interne LMS, septembre 1988.

[4] DUFFAUT P. (1981), «Stabilité des cavités souterraines $\$$, La pratique des Sols et Fondations, Ed. du Moniteur, pp. 787-823.

[5] LADANYI B. GILL D.E. (1983), "Tunnel design in a creeping rock», Proc. 5th Cong. ISRM $\propto$ Design and Performance of Underground excavations», Cambridge, pp. 19-26.

[6] LOMBARDI G., AMBERG W. (1979), «L'influence de la méthode de construction sur l'équilibre final d'un tunnel», Proc. 4th Cong. ISRM, Montreux, vol. 1, pp. 475-484.
[7] MANFROY P., NEERDAEL B., SIMON J.M., ROUSSET G. (1985), «Mesures de convergence en argile profonde», Tunnels et Ouvrages souterrains, $n^{\circ} 68$, pp. 63-71, mars-avril 1985.

[8] NGUYEN MINH D. (1986), «Modèles rhéologiques pour l'analyse du comportement différé des galeries profondes», Comptes rendus du Cong. Int. Grands Ouvrages en Souterrain, ITA/AITES, Florence, pp. 659-665.

[9] NGUYEN MINH D., ROUSSET G. (1987), "Rôle des effets à court terme sur le comportement différé des galeries souterraines», Proc. 6th ISRM Conf., Montréal.

[10] PANET M., GUELLEC P. (1974), «Contribution à l'étude du soutènement d'un tunnel à l'arrière du front de taille», Proc. 3rd ISRM Conf., Denvers, vol. II B.

[11] PIGUET J.P. (1982), «Le soutènement par cintres métalliques dans les galeries - Etude bibliographique», Document interne CERCHAR 82 11-76-1555, Laboratoire de Mécanique des Terrains, avril 1982.

[12] ROUSSET G. (1986), "Essai de fluage in situ au dilatomètre - Principe de l'essai - Eléments du Cahier des Charges», Document interne LMS, mai 1986.

[13] ROUSSET G. (1988), «Comportement mécanique des argiles profondes - Application au stockage de déchets radioactifs". Thèse de l'Ecole Nationale des Ponts et Chaussées, 4 juillet 1988.

[14] ROUSSET G., BAZARGAN B., LENAIN R. (1989), «Time dependent behavior of rocks: laboratory tests on hollow cylinders ", Proc. 30th US Symp on rock Mech. West Virginia University, Morgantown, USA, June 19-22, 1989.

[15] ROUSSET G., BUBLITZ D. (1989), «Dimensionnement des soutènements dans les roches viscoplastiques: méthode générale et expérience in situ *, Proc. Int. Symp. "Rock at great depth», Pau, France, August 28th-31st.

[16] ROUSSET G., BUBLITZ D. (1989), «Soutènement coulissant dans une argile profonde», Proc. Ing. Cong. "Progress and Innovation in Tunnelling», Toronto, Canada, sept. 9-14, 1989. 